\title{
PRODUCCIÓN ECONÓMICA DE ÁCIDO LÁCTICO UTILIZANDO RESIDUOS DE COSECHA Y JUGOS DE CAÑA DE AZÚCAR (Saccharum officinarum L.)
}

\author{
Economical production of lactic acid using sugar cane \\ (Saccharum officinarum L.) wastes and juice
}

\section{Liliana Serna Cock ${ }^{1 *}$ y Aida Rodríguez de Stouvenel ${ }^{2}$}

\section{A B S T R A C T}

Sugar cane (Saccharum officinarum L.) tops and leaves (JCG), and the juice extracted from this sugar cane, harvested with crop burning (JCL) and without crop burning (JCV), were tested as substrates for the discontinuous fermentative production of lactic acid. Anaerobic fermentations were carried out at $32^{\circ} \mathrm{C}$, adjusting $\mathrm{pH}$ at 6.0, and using Lactococcus lactis subsp. lactis isolated from the same variety of sugar cane. The effect of total sugars and nitrogen concentration on lactic acid production $(A L)$, substrate conversion $(C S)$, yield $\left(Y_{p / s}\right)$ and volumetric productivity $\left(r_{p}\right)$ were studied for the substrates mentioned above and their mixtures. Lactic acid concentrations up to $70.19 \mathrm{~g} \mathrm{~L}^{-1}, C S$ of $85.46 \%, Y_{p / s}$ of $0.88 \mathrm{~g} \mathrm{~g}^{-1}$ and $r_{p}$ of $0.97 \mathrm{~g} \mathrm{~L}^{-1} \mathrm{~h}^{-1}$ were obtained, with a total sugar concentration of $90 \mathrm{~g} \mathrm{~L}^{-1}$, from a mixture of JCG and JCV with the addition of $3 \%(\mathrm{w} / \mathrm{v})$ of yeast extract. Even without the addition of yeast extract, these mixtures performed very well as substrates for lactic acid fermentation ( $A L: 26.16 \mathrm{~g} \mathrm{~L}^{-1}, C S: 67.13 \%, Y_{p / s}: 0.69 \mathrm{~g} \mathrm{~g}^{-1}$ and $\left.r_{p}: 0.55 \mathrm{~g} \mathrm{~L}^{-1} \mathrm{~h}^{-1}\right)$; this is explained in part by the higher nitrogen content of JCG and JCV, compared to JCL. Juice extracted from cane tops and leaves, mixed with cane juice extracted from crops harvested without burning, is a cheap alternative as a raw material for the fermentative production of lactic acid.

Key words: waste products, green cane, lactic acid, cane tops, clean cane, Lactococcus lactis subsp. lactis.

\section{R E S U M E N}

Para la producción fermentativa discontinua de ácido láctico, se evaluaron como sustratos los jugos de cogollos y hojas (JCG) de la caña de azúcar (Saccharum officinarum L.) y los jugos extraídos de esta caña, cosechada con quema (JCL) y sin quema (JCV). Las fermentaciones se realizaron anaeróbicamente, a $32^{\circ} \mathrm{C}$, con ajuste de $\mathrm{pH}$ a 6,0 y usando Lactococcus lactis subsp. lactis aislado de cultivos de la misma variedad. Para los sustratos mencionados y sus mezclas se estudió el efecto de la concentración de azúcares totales y de nitrógeno sobre la producción de ácido láctico $(A L)$, la conversión de sustrato $(C S)$, el rendimiento $\left(Y_{p / s}\right)$ y la productividad volumétrica $\left(r_{p}\right)$. Se obtuvieron concentraciones de ácido láctico por encima de $70,19 \mathrm{~g} \mathrm{~L}^{-1}, C S \mathrm{de} 85,46 \%, Y_{p / s}$ de $0,88 \mathrm{~g}$ $\mathrm{g}^{-1} \mathrm{y} r_{p}$ de $0,97 \mathrm{~g} \mathrm{~L}^{-1} \mathrm{~h}^{-1}$ con una concentración de $90 \mathrm{~g} \mathrm{~L}^{-1}$ de azúcar total usando una mezcla de JCG y JCV con adición de $3 \%(\mathrm{p} / \mathrm{v})$ de extracto de levadura. Aún sin adición de extracto de levadura, estas mezclas presentaron muy buen comportamiento como sustrato de fermentación ácido láctica $\left(A L: 26,16 \mathrm{~g} \mathrm{~L}^{-1}, C S: 67,13 \%, Y_{p / s}: 0,69\right.$ $\mathrm{g} \mathrm{g}^{-1}$ y $\left.r_{p}: 0,55 \mathrm{~g} \mathrm{~L}^{-1} \mathrm{~h}^{-1}\right)$; este comportamiento se explica en parte por el mayor contenido en nitrógeno del JCG y del JCV, comparado con el JCL. El jugo extraído de las hojas y cogollos, mezclado con el jugo extraído de la caña cosechada sin quemar es una alternativa barata como materia prima para la producción fermentativa de ácido láctico.

Palabras clave: residuos, caña verde, ácido láctico, cogollos, caña limpia, Lactococcus lactis subsp. lactis.

\footnotetext{
${ }^{1}$ Universidad Nacional de Colombia, Facultad de Ingeniería, A.A. 237, Palmira, Colombia.

E-mail: 1sernac@palmira.unal.edu.co*Autor para correspondencia.

${ }^{2}$ Universidad del Valle, Facultad de Ingeniería, A.A. 25360, Cali, Colombia. E-mail: airodri@univalle.edu.co Recibido: 20 de abril de 2005. Aceptado: 7 de julio de 2005.
} 


\section{INTRODUCCIÓN}

La producción mundial de ácido láctico es de 100.000 toneladas por año (Datta et al., 1995 citado por Wang-Yu et al., 2004), con un crecimiento en la demanda del $8,6 \%$ anual, debido al potencial que tiene este monómero de producir ácido poliláctico, un polímero biodegradable con aplicaciones industriales y médicas (Lisa, 2001; Naveena et al., 2005). Para la producción biotecnológica de ácido láctico se han utilizado diferentes sustratos puros, tales como glucosa, lactosa, almidón y celulosa, sin embargo, estos sustratos son económicamente desfavorables, no sólo porque los sustratos puros son costosos y requieren la adición de fuentes nitrogenadas complejas para producir ácido láctico en un tiempo razonable, sino también porque se requiere de un pretratamiento de los polisacáridos naturales para su posible fermentación (YoungJung et al., 2004).

De acuerdo con Tejayadi y Cheryan (1995) citado por Oh et al. (2005), el costo de la materia prima en el proceso de producción de ácido láctico a partir de lactosuero y extracto de levadura con Lactobacillus bulgaricus, representa el 68\%; Akerberg y Zacchi (2000) demostraron también que el mayor costo de producción lo representa la materia prima, y Kwon et al. (2000) indican que solamente el extracto de levadura representa más del $30 \%$ de los costos. Por lo anterior, se sigue investigando activamente en la búsqueda de sustratos de fermentación láctica de bajo costo, bajo nivel de contaminantes, alta velocidad de fermentación, alto rendimiento en ácido láctico, poca formación de subproductos y disponibilidad durante todo el año.

Por otro lado, la cosecha de caña de azúcar (Saccharum officinarum L.) en verde genera $30 \mathrm{t}$ de biomasa por cada tonelada de caña cosechada; estos residuos se pican y se esparcen sobre el campo de siembra o se colocan sobre los entresurcos o calles para su descomposición. Los residuos de cosecha en verde (inmediatamente después del corte) tienen un contenido de agua de alrededor de $75 \%$ y un contenido nutricional representado en azúcares, nitrógeno, fósforo, potasio, calcio, magnesio, hierro, manganeso, cobre y zinc (Victoria et al., 2002), nutrientes que son ideales para el crecimiento microbiano.
Por lo anterior, los objetivos de este trabajo fueron evaluar los potenciales de las mezclas de jugos de cogollos y hojas, con jugo de caña de azúcar cosechado con quema, denominado jugo de caña limpio, y con jugo de caña de azúcar cosechada sin quema, denominado jugo de caña verde, como sustratos en la producción fermentativa en discontinuo de ácido láctico; además, estudiar el efecto de la adición de extracto de levadura en los jugos mencionados y determinar el isómero de ácido láctico producido.

\section{MATERIALES Y MÉTODOS}

\section{Cepa utilizada}

Se empleó una cepa de Lactococcus lactis subsp. lactis, la cual se aisló de hojas de cultivos de caña de azúcar de la variedad CC85-92 en la Hacienda La Cabaña ( $3^{\circ} 10^{\prime}$ lat. Norte, $76^{\circ} 23^{\prime}$ long. Oeste), Cauca, Colombia. Detalles del aislamiento se indicaron en Serna y Rodríguez (2006).

\section{Fermentación}

Se formularon 12 sustratos de fermentación utilizando jugo de caña limpio (JCL), jugo de caña verde (JCV), y mezclas de éstos, con jugos de cogollos y hojas (JCG). Los JCG, JCL y JCV provenían de cosechas de caña de azúcar de 12,3 meses de edad de la variedad CC85-92, cultivada en el Centro de Investigación de la Caña de Azúcar (CENICAÑA), Florida, Valle del Cauca, y en la Hacienda La Cabaña. Los cogollos y hojas de caña de azúcar cosechada en verde, los tallos de caña de azúcar cosechada con quema, y los tallos de caña de azúcar cosechada sin quema, se sometieron a molienda por separado, utilizando un molino experimental de tres masas y los jugos recolectados se transportaron bajo refrigeración.

Los tratamientos realizados se describen en el Cuadro 1. Los JCL y JCV se evaluaron con tres concentraciones de azúcares totales: 180,110 y $90 \mathrm{~g} \mathrm{~L}^{-1}$; $180 \mathrm{~g} \mathrm{~L}^{-1}$ corresponde a jugos sin modificar su concentración de azúcares iniciales, y esta concentración se disminuyó hasta 110 y $90 \mathrm{~g} \mathrm{~L}^{-1}$ mezclando el JCL y el JCV con JCG, utilizando una relación en peso JCG/JCL ó JCV de 41/69 y 47/43, respectivamente. Los sustratos de fermentación se evaluaron además en dos niveles de concentración de fuente de nitrógeno, sin adición de extracto de levadura y con adición del $3 \%(\mathrm{p} / \mathrm{v})$ de extracto de levadura. 
Cuadro 1. Definición de tratamientos y convenciones.

Table 1. Definition of treatments and conventions.

\begin{tabular}{|c|c|c|}
\hline Tratamiento & Abreviatura & Definición de la abreviatura \\
\hline 1 & JCV180-0N & $\begin{array}{l}\text { JCV sin modificar su concentración de azúcares totales y sin adición de extracto de } \\
\text { levadura. }\end{array}$ \\
\hline 2 & JCV180-3N & $\begin{array}{l}\text { JCV sin modificar su concentración de azúcares totales }+3 \%(\mathrm{p} / \mathrm{v}) \text { de extracto de } \\
\text { levadura. }\end{array}$ \\
\hline 3 & JCV110-0N & $\begin{array}{l}\text { Mezcla de JCG + JCV }(41 / 69 \mathrm{p} / \mathrm{p}) \text {. Concentración en azúcares totales de aprox. } \\
110 \mathrm{~g} \mathrm{~L}^{-1} \text { sin adición de extracto de levadura. }\end{array}$ \\
\hline 4 & JCV110-3N & $\begin{array}{l}\text { Mezcla de JCG + JCV }(41 / 69 \mathrm{p} / \mathrm{p}) \text {. Concentración de azúcares totales de aprox. } \\
110 \mathrm{~g} \mathrm{~L}^{-1}+3 \%(\mathrm{p} / \mathrm{v}) \text { de extracto de levadura. }\end{array}$ \\
\hline 5 & JCV90-0N & $\begin{array}{l}\text { Mezcla de JCG + JCV ( } 47 / 43 \mathrm{p} / \mathrm{p}) \text {. Concentración de azúcares totales de aprox. } \\
90 \mathrm{~g} \mathrm{~L}^{-1} \text { sin adición de extracto de levadura. }\end{array}$ \\
\hline 6 & JCV90-3N & $\begin{array}{l}\text { Mezcla de JCG + JCV }(47 / 43 \mathrm{p} / \mathrm{p}) \text {. Concentración de azúcares totales de aprox. } \\
90 \mathrm{~g} \mathrm{~L}^{-1}+3 \%(\mathrm{p} / \mathrm{v}) \text { de extracto de levadura. }\end{array}$ \\
\hline 7 & JCL180-0N & $\begin{array}{l}\text { JCL sin modificar su concentración de azúcares totales y sin adición de extracto de } \\
\text { levadura. }\end{array}$ \\
\hline 8 & JCL180-3N & $\begin{array}{l}\text { JCL sin modificar su concentración de azúcares totales }+3 \%(p / v) \text { de extracto de } \\
\text { levadura. }\end{array}$ \\
\hline 9 & JCL11 & $\begin{array}{l}\text { Mezcla de JCG + JCL ( } 41 / 69 \mathrm{p} / \mathrm{p}) \text {. Concentración en azúcares totales de aprox. } \\
110 \mathrm{~g} \mathrm{~L}^{-1} \sin \text { adición de extracto de levadura. }\end{array}$ \\
\hline 10 & JCL110-3N & $\begin{array}{l}\text { Mezcla de JCG + JCL }(41 / 69 \mathrm{p} / \mathrm{p}) \text {. Concentración en azúcares totales de aprox. } \\
110 \mathrm{~g} \mathrm{~L}^{-1}+3 \%(\mathrm{p} / \mathrm{v}) \text { de extracto de levadura. }\end{array}$ \\
\hline 11 & JCL90-0N & $\begin{array}{l}\text { Mezcla de JCG + JCL ( } 47 / 43 \mathrm{p} / \mathrm{p}) \text {. Concentración en azúcares totales de aprox. } \\
90 \mathrm{~g} \mathrm{~L}^{-1} \text { sin adición de extracto de levadura. }\end{array}$ \\
\hline 12 & JCL90-3N & $\begin{array}{l}\text { Mezcla de JCG + JCL }(47 / 43 \mathrm{p} / \mathrm{p}) \text {. Concentración en azúcares totales de aprox. } \\
90 \mathrm{~g} \mathrm{~L}^{-1}+3 \%(\mathrm{p} / \mathrm{v}) \text { de extracto de levadura. }\end{array}$ \\
\hline
\end{tabular}

Una vez preparados los sustratos se envasaron en erlenmeyer de $500 \mathrm{~mL}$, con un volumen de trabajo de $250 \mathrm{~mL}$ y se sometieron a esterilización por $10 \mathrm{~min}$ a $121^{\circ} \mathrm{C}$. Cuando los sustratos se encontraban a temperatura de $30^{\circ} \mathrm{C}$ aproximadamente, se inocularon con la cepa de Lactococcus lactis, la cual antes de la inoculación se adaptó a los diferentes sustratos por tres generaciones en un volumen de $10 \mathrm{~mL}$; en todos los casos se utilizó $10 \%$ de inóculo con respecto al volumen de trabajo.

Se realizaron 36 fermentaciones en condiciones anaeróbicas por $72 \mathrm{~h}$; durante el tiempo de fermentación se mantuvo el $\mathrm{pH}$ en $6 \pm 0,2$ con $\mathrm{NaOH}$ $5 \mathrm{M}$, la temperatura en $32^{\circ} \mathrm{C}$ y la velocidad de agitación en $120 \mathrm{rpm}$. Las condiciones anotadas se eligieron basadas en estudios previos de Serna y Rodríguez (2006). Después de realizar el análisis estadístico de los resultados de las 36 fermentaciones, se realizaron tres fermentaciones adicionales a escala de $1 \mathrm{~L}$, para esto, se usó el sustrato que presentó la mayor eficiencia y se emplearon las mismas condiciones descritas anteriormente.

\section{Método analítico}

Se tomaron muestras de líquidos de fermentación a las $0,2,4,6,9,12,24,48$ y $72 \mathrm{~h}$, se centrifugaron a $5.000 \mathrm{~g}$ por $10 \mathrm{~min}$ y luego se filtraron con filtros de $0,45 \mu$ (Millipore, HVLP02500, Billerica, USA). Las concentraciones de azúcares y de ácido láctico se midieron en los sobrenadantes por cromatografía líquida de alta precisión HPLC (Hitachi L6000A, integrador D-2500, Tokyo, Japón) equipada con una columna para determinación de ácidos y azúcares (Aminex HPX 87H, $300 \mathrm{~mm}$ ), utilizando como fase móvil ácido sulfúrico $0,005 \mathrm{M}$. La biomasa se calculó a partir de datos de densidad óptica a $540 \mathrm{~nm}\left(\mathrm{DO}_{540}\right)$ utilizando un espectrofotómetro (Milton Roy 401, Rochester, USA). Para establecer una correlación lineal entre los valores de densidad óptica y los valores de biomasa, se midieron las densidades ópticas y el peso seco de las células, a las $0,2,4,6,9,12,24,48$ y 72 h de fermentación. Estas mediciones se usaron por triplicado. La correlación estimada se usó para convertir todos los valores de $\mathrm{DO}_{540}$ a concentración de biomasa; sin embargo, para corroborar los resultados, al final de cada una de las fermentaciones, se tomaron muestras de caldo de fermentación 
para determinar la biomasa láctica y la densidad óptica. El pH se midió utilizando un pHmetro (Orion 710A, Cambridge, USA).

Los porcentajes de conversión de sustrato $(C S)$, los rendimientos en producto $\left(Y_{p / s}\right) \mathrm{g} \mathrm{g}^{-1}, \mathrm{y}$ la productividad volumétrica $\left(r_{p}\right)$, se calcularon mediante las siguientes expresiones:

$$
\begin{aligned}
& \mathrm{CS}=\frac{100 *\left(S_{0}-S\right)}{S_{0}} \\
& Y_{p / s}=\frac{P}{S_{0}-S} \\
& r_{p}=\frac{d_{p}}{d_{t}}
\end{aligned}
$$

donde, $S_{0}$ : concentración de azúcares totales iniciales $\left(\mathrm{g} \mathrm{L}^{-1}\right) ; S$ : concentración de azúcares totales finales (g $\left.\mathrm{L}^{-1}\right)$, hasta el tiempo en que $P$ es máximo; $P$ : concentración máxima de ácido láctico $\left(\mathrm{g} \mathrm{L}^{-1}\right)$; y $t$ : tiempo de fermentación. La productividad volumétrica, $r_{p}$, se calculó derivando las ecuaciones que ajustan los datos cinéticos.

A los sustratos de fermentación en condiciones iniciales, se les midió azúcares totales por HPLC, nitrógeno total mediante el método de Kjeldahl (AOAC, 1980), fósforo por colorimetría utilizando el método del cloruro estañoso (la lectura se realizó a $690 \mathrm{~nm}$ en un espectrofotómetro (Shimazu UV 160 PC, Tokio, Japón); y potasio, calcio y magnesio mediante absorción atómica (Perkin Elmer 3110, Norwalk, USA) empleando llama de óxido nitroso-acetileno. La cepa se identificó en API 50 CHL y la identificación isomérica del ácido láctico producido en todos los sustratos se realizó mediante bioanálisis enzimático D-ácido láctico/Lácido láctico.

\section{Reactivos}

Se utilizaron productos grado reactivo (Sigma Chemical Co., St. Louis, USA).

\section{Diseño estadístico}

Para evaluar el potencial como sustratos de fermentación láctica, de los JCL, JCV y las mezclas de JCG con JCL y JCV, se utilizó un diseño factorial de $2 * 3 * 2$ con tres repeticiones, así: tipo de jugo de caña de azúcar con dos niveles: mezclas de JCL con JCG y mezclas de JCV con JCG; concentración de azúcar total con tres niveles: 180, 110 y $90 \mathrm{~g} \mathrm{~L}^{-1}$; concentración de nitrógeno total con dos niveles: jugos sin modificar el contenido en nitrógeno ( $\sin$ adición de extracto de levadura) y modificando el contenido de nitrógeno (con adición de $3 \% \mathrm{p} / \mathrm{v}$ de extracto de levadura). Las diferentes concentraciones en azúcares totales se obtuvieron mediante mezclas de los JCV y JCL con JCG, como se describió en el Cuadro 1.

Las variables de respuesta fueron: concentración en ácido láctico $(A L)$, conversión de sustrato $(C S)$, rendimiento en producto $\left(Y_{p / s}\right)$ y productividad volumétrica $\left(r_{p}\right)$.

Los datos se analizaron mediante análisis de varianza (ANDEVA) para tres factores con efectos fijos. Las diferencias entre los pares de medias de las variables que mostraron diferencias significativas se analizaron mediante la prueba de diferencia significativa honesta de Tukey (DSH). El diseño se analizó con un nivel de confianza del 95\%.

\section{RESULTADOS Y DISCUSIÓN}

Las cinéticas de formación de biomasa, formación de producto y consumo de sustrato de los 12 tratamientos, y los parámetros cinéticos $A L, C S, Y_{p / s}$ y $r_{p}$, pueden observarse en la Figura 1 y Cuadro 2 , respectivamente. En todos los sustratos se produjo $\mathrm{L}(+)$ ácido láctico. Los sustratos que presentaron las más altas concentraciones en ácido láctico y los más altos rendimientos en producto, mostraron también las más altas productividades (Cuadro 2).

El ANDEVA para las tres variables independientes, tipo de jugo de caña (A), concentración de azúcar total (B) y concentración de extracto de levadura (C), y las interacciones $\mathrm{B} * \mathrm{C}$ y $\mathrm{A} * \mathrm{~B} * \mathrm{C}$ mostraron $\mathrm{P}<0,05$ para la concentración de ácido láctico. El ANDEVA para concentración de ácido láctico se puede ver en el (Cuadro 3).

Se obtuvieron correlaciones lineales para la concentración de ácido láctico en función de la concentración de azúcares totales y de la concentración de extracto de levadura; ecuaciones de regresión (1) para $A L$ con JCV y ecuación de regresión (2) para $A L$ con JCL, con coeficientes de correlación de 0,95 y 0,97 , respectivamente. 
a)
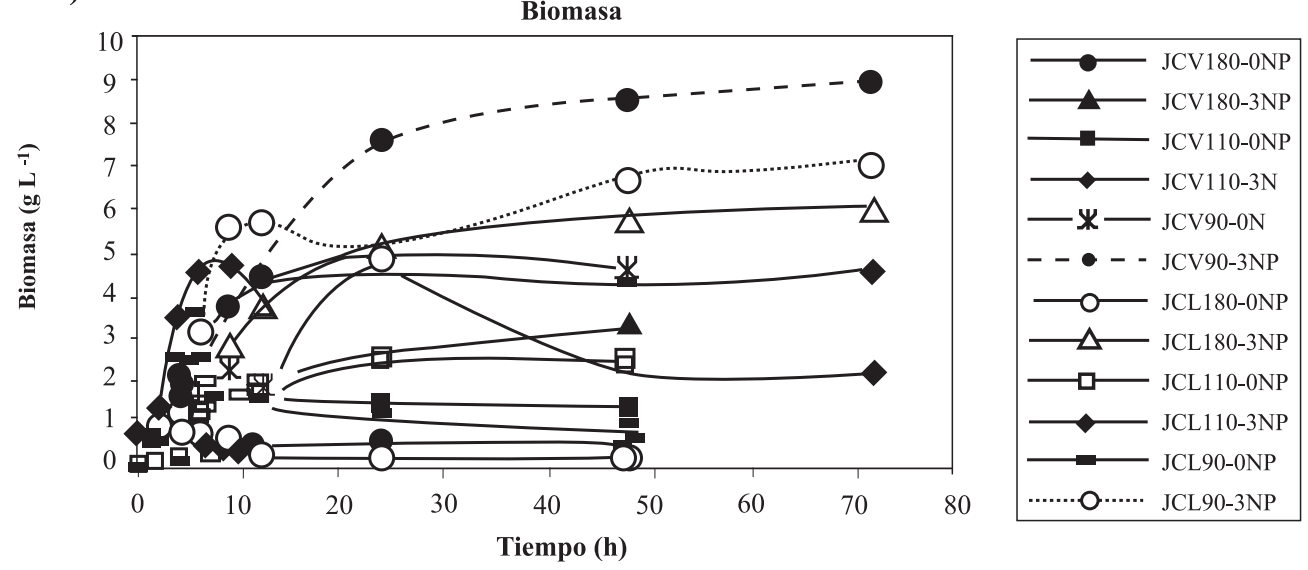

b)
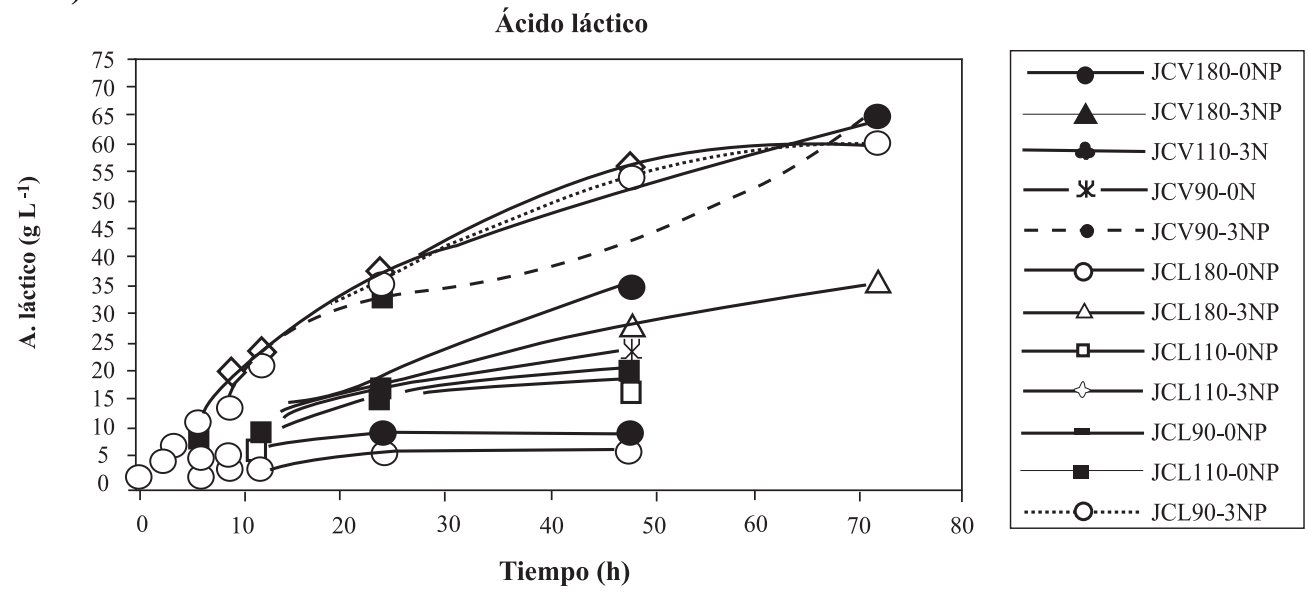

c)
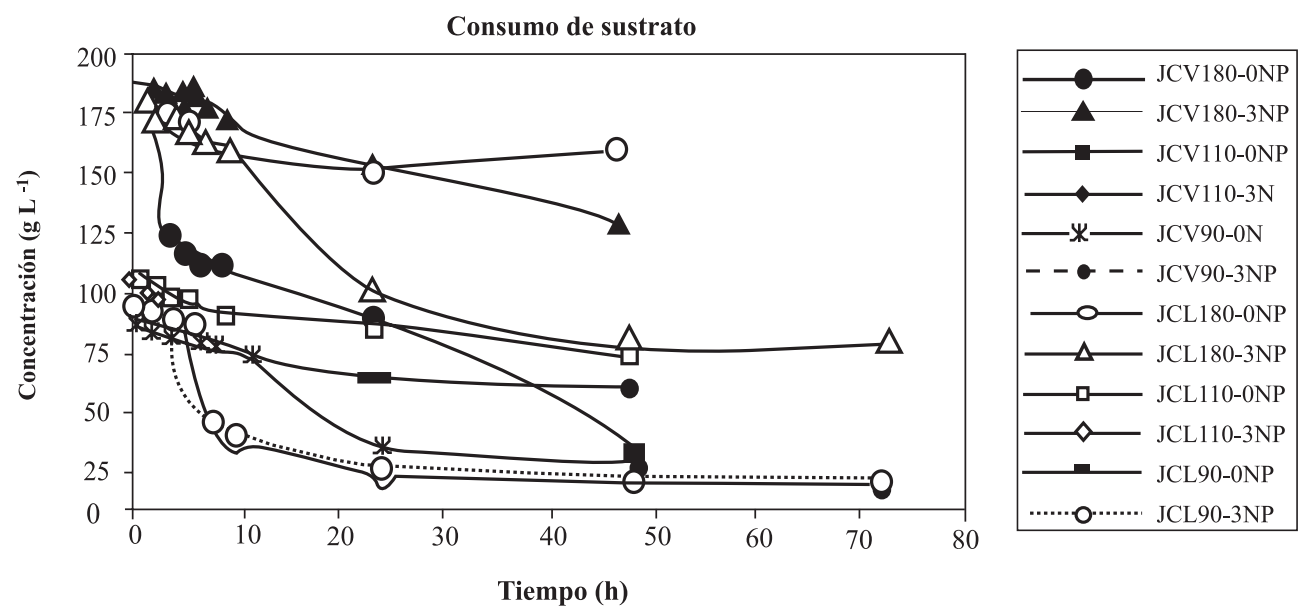

Figura 1. a) Cinética de formación de biomasa; b) cinética de formación de producto; c) cinética de consumo de sustrato.

Figure 1. a) Kinetic of biomass formation; b) kinetic of product formation; c) kinetic of substrate consumption. 


$$
\begin{aligned}
& A L=50,2-0,254 A T+13,9 E L(1) \\
& A L=48,6-0,251 A T+13,3 E L(2)
\end{aligned}
$$

donde: $A L=$ concentración de ácido láctico $\left(\mathrm{g} \mathrm{L}^{-1}\right)$; $A T=$ concentración de azúcares totales $\left(\mathrm{g} \mathrm{L}^{-1}\right) ; \mathrm{y}$ $E L=$ concentración de extracto de levadura $(\% \mathrm{p} / \mathrm{v})$.

Puede notarse que tanto para JCV (Ecuación 1) como para JCL (Ecuación 2) el extracto de levadura tiene un fuerte efecto directo sobre la concentración de ácido láctico, por lo que su aumento implica un incremento en la concentración de ácido láctico. Por otra parte, la concentración de azúcares totales muestra un efecto mucho menor e inverso sobre la concentración de ácido láctico, lo que significa que un aumento en la concentración de azúcares produce una ligera disminución en la concentración de ácido láctico.

En la Figura 2 se presenta el comportamiento de los datos experimentales frente a los datos calculados mediante las ecuaciones (1) y (2); se aprecia que los datos predichos se ajustan muy bien a una línea de $45^{\circ}$.

El tipo de jugo, la concentración de azúcares totales, la concentración de extracto de levadura y todas las interacciones presentaron diferencias significativas en el ANDEVA para $C S$ y $r_{p}$.

La concentración de azúcares totales, la concentración de extracto de levadura y las interacciones $\mathrm{A} * \mathrm{~B}$, $\mathrm{A} * \mathrm{C}$ y $\mathrm{B} * \mathrm{C}$ mostraron $\mathrm{P}<0,05$ para el rendimiento.

El error experimental en todos los casos presentó un valor de $\mathrm{P}>0,05$, lo que indica que no hubo diferencia entre las repeticiones.

Las diferencias significativas encontradas entre los tratamientos se explican por las diferencias en la concentración de nitrógeno total entre ellos (Cuadro 2), porque el JCG- $0 \mathrm{~N}$ contiene 3,2 veces más fósforo $(0,032 \%), 2,5$ veces más potasio $(0,54 \%)$ y 1,3 veces más magnesio $(0,023 \%)$ que el JCL180$0 \mathrm{~N}, \mathrm{y}$ además, porque el JCG- $0 \mathrm{~N}$ contiene, 1,1 veces más fósforo, 4,7 veces más potasio y 1,6 veces más magnesio que el JCV180-0N. Fuera de la fuente de carbono, el contenido de estos micronutrientes es de vital importancia para las reacciones enzimáticas y la síntesis de paredes y membranas celulares en el crecimiento de L. lactis y por ende para la producción de ácido láctico, dado que este metabolito está asociado al metabolismo energético, 
Cuadro 3. ANDEVA para concentración de ácido láctico.

Table 3. ANOVA for lactic acid concentration.

\begin{tabular}{lcccrrr}
\hline Fuente & $\begin{array}{c}\text { Grados } \\
\text { de libertad }\end{array}$ & $\begin{array}{c}\text { Suma } \\
\text { de cuadrados }\end{array}$ & Varianzas & $\begin{array}{c}\text { Cuadrado } \\
\text { medio }\end{array}$ & F & P \\
\hline Tipo de jugo (A) & 1 & 39,4 & 39,4 & 39,4 & 19,24 & 0,0001 \\
Concentración de azúcar total (B) & 2 & 34,393 & 34,393 & $1.719,6$ & 840,19 & 0,0002 \\
Extracto de levadura (C) & 1 & $15.018,9$ & $15.018,9$ & $15.018,9$ & $7.338,01$ & 0,0001 \\
A*B & 2 & 13,7 & 13,7 & 6,9 & 3,36 & 0,052 \\
$A^{*} \mathrm{C}$ & 1 & 6,6 & 6,6 & 6,6 & 3,22 & 0,085 \\
$\mathrm{~B}^{*} \mathrm{C}$ & 2 & 526,2 & 526,2 & 263,1 & 128,54 & 0,000 \\
A B $^{*} \mathrm{C}$ & 2 & 41,2 & 41,2 & 20,6 & 10,06 & 0,001 \\
Error & 24 & 49,1 & 49,1 & 2 & & \\
Total & 35 & $19.134,4$ & & & & \\
\hline
\end{tabular}

a)

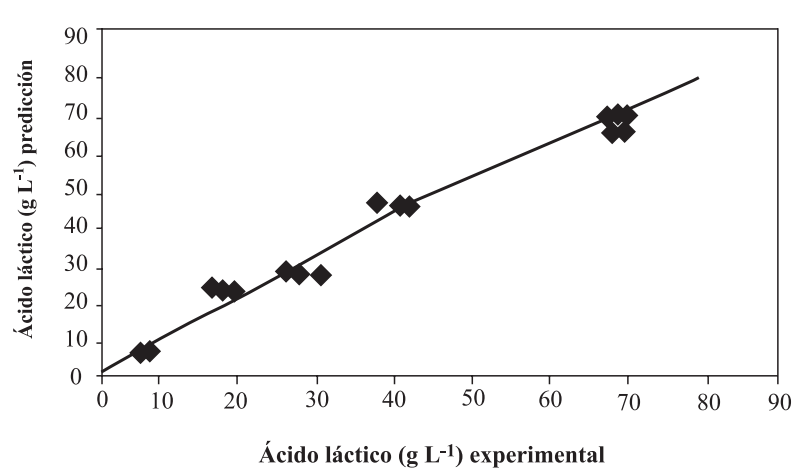

b)

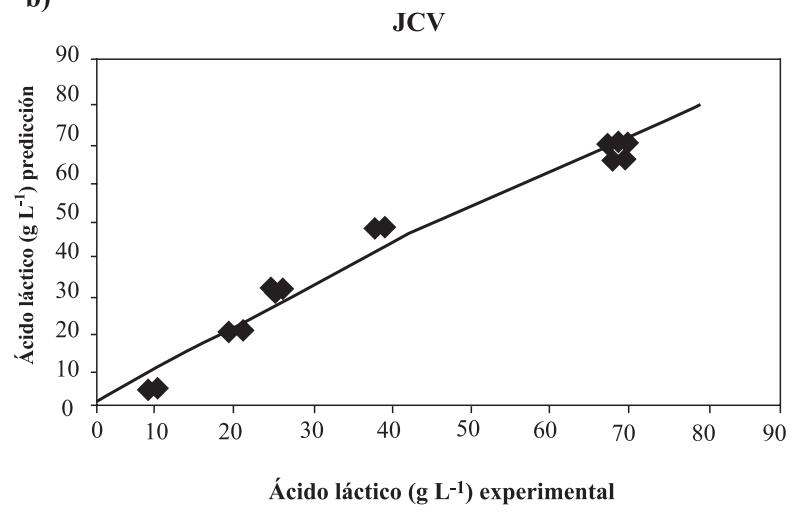

Figura 2. Comportamiento del modelo de predicción de la concentración de ácido láctico. a) En jugo de caña limpio (JCL); b) en jugo de caña verde (JCV).

Figure 2. Acid lactic concentration model prediction performance. a) Juice extracted from sugar cane harvested with crop burning (JCL); b) juice extracted from sugar cane harvested without crop burning (JCV).

tema discutido ampliamente por Niel y Hahn-Hägerdal (1999).

El comportamiento de los sustratos a los cuales no se les adicionó extracto de levadura, sugiere que tanto en los jugos de cogollos como en los jugos de caña se encuentran, además de los micronutrientes señalados, factores de crecimiento, aminoácidos y ácidos grasos, considerados esenciales para el crecimiento y la producción de ácido láctico por $L$. lactis aislados de ambientes no lácteos, tales como ácido glutámico, valina, metionina, histidina, tiamina, riboflavina, acetato y ácido oléico, como plantean Godon et al. (1993). Asimismo, como estos sustratos provienen de material vegetal, el contenido de ácidos nucleicos proveen de citosina, adenina, guanina y uracilo, bases que tienen un conocido efecto estimulador del crecimiento de $L$. lactis (Cocaign-Bousquet et al., 1995).
$A L, Y_{p / s}$ y $r_{p}$ obtenidas con JCV90-3N, JCL90-3N, JCL110-3N y JCL110-3N, fueron superiores a los resultados obtenidos por Roukas y Kotzekidou (1996, 1998), Akerberg et al. (1998), Ohashi et al. (1999), quienes investigaron con Lactococcus lactis la producción de ácido láctico en fuentes de carbono diferentes a glucosa, tales como xilosa, lactosuero, harina de trigo (Triticum sativum L.) hidrolizada y no hidrolizada, y melazas respectivamente. Se exceptúan los resultados de Hofvendahl y Hahn-Hägerdal (1997), quienes trabajaron con harina de trigo y obtuvieron $A L, Y_{p s s} \mathrm{y} r_{p}$ mayores que los obtenidos en este estudio, y los resultados de Hofvendahl (1998) y Hofvendahl et al. (1999), quienes obtuvieron $r_{p}$ mayores en harina de trigo y maltosa. De igual manera al comparar $A L, Y_{p / s}$ y $r_{p}$ obtenidas con JCV90-3N, JCL90-3N, JCL110-3N y JCL110-3N con los resultados de investigaciones en los cuales se utilizó L. lactis en glucosa pura, se 
puede observar que en sustrato puro, todas las $A L$ obtenidas por Ishizaki y Vonktaveesuk (1996), Kwon et al. (1996), Planas et al. (1996), Hofvendahl (1998), Akerberg et al. (1998), Ohashi et al. (1999) fueron menores a las concentraciones obtenidas en JCV90-3N. Para las mismas referencias, $r_{p}$ fueron mayores a las reportadas con JCV90-3N, y $Y_{p / s}$ fue variable.

El extracto de levadura se constituyó en un nutriente esencial para la producción de ácido láctico, ya que en su ausencia la producción fue menos de la mitad de la producción máxima en $48 \mathrm{~h}$, pero no fue esencial para la producción de biomasa (Cuadro 2). El mejor comportamiento que se observó en los experimentos con adición de extracto de levadura se debe, además, al contenido de vitaminas en el extracto de levadura y a que los aminoácidos presentes están en forma libre (Benthin y Villadsen, 1996); estos nutrientes son cruciales en las etapas primarias de fermentaciones lácticas (Hujanen y Linko, 1994).

Debido a que la fuente de nitrógeno es el nutriente limitante, se requiere dirigir la investigación en el enriquecimiento de estos sustratos con otras fuentes de nitrógeno más baratas, obtenidas, por ejemplo, de residuos industriales como agua de cocimiento de maíz (Zea mays L.) o biomasa de levadura, los cuales han sido utilizados con éxito por Rivas et al. (2004); esto permitiría continuar la fermentación hasta agotar la fuente de carbono.

Si se comparan las concentraciones en ácido láctico obtenidas con L. lactis a partir de los sustratos estudiados, con los resultados de investigaciones donde se emplearon otras bacterias ácido lácticas en residuos de cosecha o sustratos diferentes a glucosa y lactosa puros, se encuentra que las concentraciones en ácido láctico que se lograron con JCG, JCV y JCL, son mayores que las obtenidas por Xiaodong et al. (1997) en $36 \mathrm{~h}$ de fermentación, quienes encontraron a partir de $10 \mathrm{~g} \mathrm{~L}^{-1}$ de almidón, trigo (Triticum aestivum L.), arroz (Oryza sativa L.), yuca (Manihot sculenta Crantz) y papa (Solanum tuberosum L.), concentraciones de ácido láctico de 10,$05 ; 7,82 ; 7,85 ; 4,72$ y $4,42 \mathrm{~g} \mathrm{~L}^{-1}$, respectivamente. Oda et al. (1997) reportan también concentraciones y rendimientos mucho más bajos, $0,13 \mathrm{~g} \mathrm{~L}^{-1}$ y $47,2 \%$ en $72 \mathrm{~h}$ de fermentación utilizando corteza de pan adicionada de agua de cocimiento de maíz; Garde et al. (2000) utilizando jugos de plan- tas verdes como trébol (Trifolium spp.) y alfalfa (Medicago sativa L.) obtuvieron concentraciones de $13 \mathrm{~g} \mathrm{~L}^{-1}$ con Lactobacillus salivarius. Bulut et al. (2004) consiguieron concentraciones máximas de $58 \mathrm{~g} \mathrm{~L}^{-1}$ con algarroba (Ceratonia siliqua L.) y Rhizopus oryzae. Naveena et al. (2005) con Lactobacillus amylophilus obtuvieron 2,3 g de ácido láctico por cada $10 \mathrm{~g}$ de salvado de trigo fermentado, y Oh et al. (2005) en harina de cebada (Hordeum vulgare L.) con Enterococcus faecalis lograron tan sólo $38 \mathrm{~g} \mathrm{~L}^{-1}$ de ácido láctico.

Los ensayos realizados en JCV90-3N presentaron $A L$ de $70,19 \mathrm{~g} \mathrm{~L}^{-1}, C S$ de $85 \%, Y_{p / s}$ de $0,88 \mathrm{~g} \mathrm{~g}^{-1}$ y $r_{p}$ de $0,97 \mathrm{~g} \mathrm{~L}^{-1} \mathrm{~h}^{-1}$; estos resultados no fueron estadísticamente diferentes a los obtenidos con $250 \mathrm{~mL}$ de JCL90-3N. Si se comparan estos resultados con los obtenidos por Serna y Rodríguez (2006), se encuentra que utilizando JCV90-3N se puede obtener $54 \%$ más ácido láctico y $28,4 \%$ más de rendimiento en producto que cuando se utiliza la misma cepa en medio de cultivo comercial ideal para bacterias ácido lácticas, caldo De Man-Rogosa-Sharpe (MRS).

Los costos de producción del ácido láctico dependen de la materia prima y del proceso de fermentación y recuperación que se utilice. Según Tejayadi y Cheryan (1995) la materia prima puede representar hasta el $68 \%$ de los costos totales de producción de ácido láctico, y según Akerberg y Zacchi (2000) los costos operacionales que incluyen el costo de la materia prima constituyen cerca del $80 \%$; en consecuencia y dado que el jugo de cogollos y hojas (componente mayoritario en los sustratos de fermentación investigados) se obtuvieron de un residuo de cosecha inutilizado, se puede concluir que éste podría constituirse en materia prima barata con alto rendimiento y disponibilidad durante todo el año para la producción económica de $\mathrm{L}(+)$ ácido láctico.

Utilizando JCV90-3N en producción en discontinuo y separación tradicional y asumiendo rendimientos de $0,88 \mathrm{~g} \mathrm{~g}^{-1} \mathrm{y}$ concentraciones de $70 \mathrm{~g} \mathrm{~L}^{-1}$, la producción de ácido láctico sería del orden de un dólar por kilogramo, esto representa un 50\% menos de los costos de producción que cuando se utiliza glucosa pura. La apreciación anterior es comparable con los conceptos de Sreenath et al. (2001), quienes empleando residuos de cosecha de alfalfa para producir ácido láctico concluyeron que éstos 
pueden utilizarse como sustrato de fermentación para producción de ácido láctico a gran escala; y con los conceptos de Garde et al. (2000) y Anderson y Kiel (2000), quienes señalan que con jugos de plantas verdes se pueden obtener mejores rendimientos en ácido láctico, que utilizando MRS, un medio comercial para bacterias ácido lácticas.

En 1986, Brizuela et al. (1986) concluyeron que el jugo de cogollos era un sustrato apropiado para la propagación de hongos y levaduras. Los resultados de este trabajo confirman además que los cogollos y hojas constituyen un buen sustrato de crecimiento incluso para las exigentes bacterias ácido lácticas, ya que se lograron obtener concentraciones hasta de 4,77 $\mathrm{g} \mathrm{L}^{-1}$ de células sin la adición de extracto de levadura, y hasta $8,86 \mathrm{~g} \mathrm{~L}^{-1}$ de células cuando los jugos se adicionaron con esta fuente de nitrógeno.

\section{CONCLUSIONES}

Las mezclas de JCG con JCL y JCV, son sustratos con muy buen potencial para ser utilizados en la producción de $\mathrm{L}(+)$ ácido láctico, aún sin la adi- ción de extracto de levadura. De las mezclas evaluadas, cuando se utiliza como sustrato JCV90-3N, se pueden obtener las más altas concentraciones en ácido láctico, las más altas conversiones de sustrato, los mayores rendimientos en producto y las mejores productividades volumétricas. El uso de este sustrato podría disminuir los costos operacionales en más del 50\%, comparado con el uso de sustratos puros, y daría además un valor agregado muy interesante a este residuo que se deja perder en el campo.

La concentración de ácido láctico en mezclas de JCG con JCV y con JCL se puede correlacionar linealmente con la concentración de azúcares totales y con la concentración de extracto de levadura con coeficientes de regresión de 0,95 y 0,97 respectivamente.

\section{RECONOCIMIENTO}

Las autoras agradecen al Centro de Investigación de la Caña de Azúcar (CENICAÑA) y al Ingenio La Cabaña por el aporte valioso en esta investigación.

\section{LITERATURA CITADA}

Akerberg, C., K. Hofvendahl, G. Zacchi, and B. HahnHägerdal. 1998. Modeling the influence of $\mathrm{pH}$, temperature, glucose and lactic acid concentrations on the kinetics of lactic acid production by Lactococcus lactis spp. lactis ATCC 19435 in whole wheat flour. Appl. Microbiol. Biotechnol. 49:682-690.

Akerberg, C., and G. Zacchi. 2000. An economic evaluation of the fermentative production of lactic acid from wheat flour. Bioresource Technol. 75:119-126.

Anderson, M., and P. Kiel. 2000. Integrated utilization of green biomass in the green biorefinery. Ind. Crops Prod. 11:129-137.

AOAC. 1980. Oficial methods of analysis. $13^{\text {th }}$ ed. 1018 p. Association of Official Agricultural Chemist, Washington DC., USA.

Benthin, S., and J. Villadsen. 1996. Amino acid utilization by Lactococcus lactis subsp. cremoris FDI during growth on yeast extract or casein peptone. J. Appl. Bacteriol. 80:65-72.

Brizuela, M., P. Sferrano, F. Campos, R. Rodríguez, y S. Armenteros. 1986. Obtención de biomasa a partir de jugo de cogollos de caña. Revista ICIDCA sobre los Derivados de la Caña de Azúcar (Cuba) 20(3):1-6.
Bulut, S., M. Elibol, and D. Ozer. 2004. Effect of different carbon sources on $\mathrm{L}(+)$-lactic acid production by Rhizopus oryzae. Biochem. Eng. J. 21:33-37.

Cocaign-Bousquet, M., C. Garrigues, L. Novak, N.D. Lindley, and P. Loubiere. 1995. Rational development of a simple synthetic medium for the sustained growth of Lactococcus lactis. J. Appl. Bacteriol. 79:108-116.

Datta, R., S. Tsai, P. Bonsignore, S. Moon, and J. Frank. 1995. Technological and economic potential of poly (lactic acid) and lactic acid derivatives. FEMS Microbiol. Rev. 16:221-231.

Garde, A., A. Schmidt, G. Jonsson, M. Andersen, A.B. Thomsen, B.K. Ahring, and P. Kiel. 2000. Agricultural crops and residuals as a basis for polylactate production in Denmark. p. 50-55. In Weber, C.J. (ed.). Conference proceedings. The Food Biopack Conference, 27-29 August. Royal Veterinary and Agricultural University, Copenhagen. Denmark.

Godon, J-J., C. Delorme, J. Bardowski, M-C. Chopin, S.D. Ehlich, and P. Renault. 1993. Gene inactivación in Lactococcus lactis. I. Branched-chain amino acid biosynthesis. J. Bacteriol. 175:4383-4390. 
Hofvendahl, K. 1998. Fermentation of wheat starch hydrolysate by Lactococcus lactis: factors affecting product formation. Ph.D. Thesis. Lund University, Lund, Sweden.

Hofvendahl, K., C. Akerberg, G. Zacchi, and B. HahnHägerdal. 1999. Simultaneous enzymatic wheat starch saccharification and fermentation to lactic acid by Lactococcus lactis. Appl. Microbiol. Biotechnol. 52:163-169.

Hofvendahl, K., and B. Hahn-Hägerdal. 1997. L-lactic acid production from whole wheat flour hydrolysate using strains of Lactobacilli and Lactococci. Enzyme Microb. Technol. 20:301-307.

Hujanen, M., and Y-Y. Linko. 1994. Optimization of L(+)lactic acid production employing statistical experimental design. Biotechnol. Technol. 8:325-330.

Ishizaki, A., and P. Vonktaveesuk. 1996. Optimization of substrate feed for continuous production of lactic acid by Lactococcus lactis IO-1. Biotechnol. Lett. 18:1113-1118.

Kwon, Y.J., R. Kaul, and B. Mattiasson. 1996. Extractive lactic acid fermentation in poly (ethyleneimine)based aqueous two-phase system. Biotechnol. Bioeng. 50:280-290.

Kwon, S., P.CH. Lee, E.G. Lee, Y.K. Chang, and N. Chang. 2000. Production of lactic acid by Lactobacillus rhamnosus with vitamin-supplemented soybean hydrolysate. Enzyme Microb. Tech. 26:209215.

Lisa, J. 2001. Lactic acid outlook up as poly lactide nears market. Chem. Market Rep. 26:14.

Naveena, B., M. Altaf, K. Bhadrayya, S. Madhavendra, and G. Reddy. 2005. Direct fermentation of starch to $\mathrm{L}(+)$ lactic acid in SSF by Lactobacillus amylophilus GV6 using wheat bran as support and substrate: medium optimization using RSM. Process Biochem. 40:681-690.

Niel, E., and B. Hahn-Hägerdal. 1999. Nutrient requirements of Lactococci in defined growth media. Appl. Microbiol. Biotechnol. 52:617-627.

Oda, Y., B. Park, K. Moon, and K. Tonomura. 1997. Recycling of bakery wastes using an amylolytic lactic acid bacterium. Bioresource Technol. 60:101-106.

Oh, H., Y. Wee, J. Yun, S. Han, S. Jung, and H. Ryu. 2005. Lactic acid production from agricultural resources as cheap raw materials. Bioresource Technol. 96:1492-1498.

Ohashi, R., T. Yamamoto, and T. Suzuki. 1999. Continuous production of lactic acid from molasses by perfusion culture of Lactococcus lactis using a stirred ceramic membrane reactor. J. Biosci. Bioeng. 87:647-654.
Planas, J., D. Lefebvre, F. Tjerneld, and B. HahnHägerdal. 1996. Analysis of phase composition in aqueous two-phase systems using a two-column chromatographic method: application to lactic acid production by extractive fermentation. Biotechnol. Bioeng. 54:303-311.

Rivas, B., A. Moldes, J. Domínguez, and J. Parajo. 2004. Development of culture media containing spent yeast cell of Debaryomyces hanseii and corn step liquour for lactic acid production with Lactobacillus rhamnosus. Int. J. Food Microbiol. 97:93-98.

Roukas, T., and P. Kotzekidou. 1996. Continuous production of lactic acid from deproteinized whey by coimmobilized Lactobacillus casei and Lactococcus lactis cells in a packed-bed reactor. Food Biotechnol. 10:231-242.

Roukas, T., and P. Kotzekidou. 1998. Lactic acid production from deproteinized whey by mixed cultures of free and coimmobilized Lactobacillus casei and coimmobilized Lactobacillus casei and Lactococcus lactis cells using fedbatch culture. Enzyme Microb. Technol. 22:199-204.

Serna, L., and A. Rodríguez. 2006. Lactic acid production by a strain of Lactococcus lactis subsp. lactis isolated from sugar cane plants. Elect. J. Biotechnol. 9:40-45.

Sreenath, H., R. Koegel, A. Moldes, and R. Straub. 2001. Lactic acid production from agriculture residues. Biotechnol. Lett. 23:179-184.

Tejayadi, S., and M. Cheryan. 1995. Lactic acid from cheese whey permeate. Productivity and economics of a continuous membrane bioreactor. Appl. Microbiol. Biotechnol. 43:242-248.

Victoria, J., A. Amaya, H. Rangel, C. Viveros, C. Cassalett y J. Carbonell, et al. 2002. Características agronómicas y de productividad de la variedad Cenicaña Colombia (CC). CENICAÑA Serie Técnica 30. p. 85-92. Cali, Valle, Colombia.

Wang-Yu, T., F. Xiang-Yang, L. San-Mok, Y. Jie, L. JianWen, W. Dong-Zhi, and K. Yoon-Mo. 2004. Purification of $\mathrm{L}(+)$-lactic acid from fermentation broth with paper sludge as a cellulosic feedstock using weak anion exchanger Amberlite IRA-92. Biochem. Eng. J. 18:89-96.

Xiaodong, W., G. Xuan, and S. Rakshit. 1997. Direct fermentative production of lactic acid on cassava and other starch substrates. Biotechnol. Lett. 19:841-843.

Young-Jung, W., K. Jin-Nam, Y. Jong-Sun, and R. HwaWon. 2004. Utilization of sugar molasses for economical $\mathrm{L}(+)$-lactic acid production by batch fermentation of Enterococcus faecalis. Enzyme Microb. Technol. 35:568-573. 